\title{
REPRESENTATION OF BOREL ISOMORPHISM BY A PROBABILITY MEASURE
}

\author{
R. M. SHORTT
}

(Communicated by R. Daniel Mauldin)

\begin{abstract}
A theorem of Chuaqui on the representation of equivalence relations by probability measures is applied to Borel isomorphism. Using a result of Cenzer and Mauldin, we show that under $V=L$, the isomorphism types of analytic sets cannot be linearly ordered.
\end{abstract}

Let $X$ and $Y$ be topological spaces. We indicate their Borel isomorphism types by $t(X)$ and $t(Y)$, writing $t(X) \leq t(Y)$ in case there is a Borel-isomorphism of $X$ onto a Borel subset of $Y$. The usual Cantor-Schroeder-Bernstein construction shows that $\leq$ is a partial order on the class of all types [2, Theorem 2.5]. Given $n=1,2, \ldots, \omega$ and $t=t(X)$, define $n t=t(n X)$, where $n X$ is the topological sum of $n$ copies of $X$. Put

$$
\sec (t)=\{t(B): B \text { Borel subset of } X\} .
$$

Then $\sec (t)$, under $\leq$, is a partially ordered set with largest element $t$.

A topological space is Polish if it is separable and completely metrizable. If $A$ and $B$ are Borel subsets of a Polish space $P$, then $t(A)=t(B)$ if and only if $A$ and $B$ have the same cardinality $[5,8.3 .6]$. It follows that for $t=t(S), S$ an infinite Borel subset of $P$, we have $t=\omega t$.

A metric space is analytic if it is a continuous image of a Polish space. There is an analytic space $U$ such that $A N=\sec (t(U))$ contains the isomorphism types of all analytic spaces: $U$ is a "universal" analytic set $[5,8.2 .16]$. The structure of the poset $(A N, \leq)$ has been the object of much study. See [1] for references.

Lusin [7] discovered that there are at least two uncountable analytic isomorphism types, viz. $t(\mathbf{R})$ and $t(U)$. Harrington and Steel $[6,8]$ showed that there are exactly two if and only if all analytic games are determined, e.g. if there is a measurable cardinal. In particular, this implies that $(A N, \leq)$ is linearly ordered. In [1], Cenzer and Mauldin proved that under $V=L,|A N|=2^{\omega}$ and there is an uncountable analytic type $t$ with $n t \neq m t$ for $n \neq m$ positive integers. In particular, it was shown that $t \neq \omega t$. Using a result of Chuaqui, we will show how this phenomenon excludes the possibility that $\leq$ linearly orders $A N$.

Let $(X, \mathscr{B})$ be a measurable space and let $\sim$ be an equivalence relation on $\mathscr{B}$. Then $\sim$ is refining if whenever $A \sim B$ are sets in $\mathscr{B}$ such that

$$
A=A_{1} \cup A_{2}, \quad A_{1} \cap A_{2}=\varnothing, \quad A_{1}, A_{2} \in \mathscr{B},
$$

Received by the editors July 6, 1987 and, in revised form, September 24, 1987.

1980 Mathematics Subject Classification (1985 Revision). Primary 04A15; Secondary 28A05, $54 \mathrm{H} 05$.

Key words and phrases. Analytic set, Borel isomorphism, Borel set. 
then there are sets $B_{1}, B_{2}$ in $\mathscr{B}$ such that

$$
B=B_{1} \cup B_{2}, \quad B_{1} \cap B_{2}=\varnothing, \quad A_{1} \sim B_{1}, \quad A_{2} \sim B_{2} .
$$

Say that $\sim$ is countably additive if whenever $A_{1}, A_{2}, \ldots$ and $B_{1}, B_{2}, \ldots$ are each pair-wise disjoint sequences of sets in $\mathscr{B}$ with $A_{n} \sim B_{n}$ for $n \geq 1$, then $\bigcup A_{n} \sim$ $\bigcup B_{n}$. A set $A \in \mathscr{B}$ is negligible for $\sim$ if there is a pairwise disjoint sequence $A_{1} A_{2} \ldots$ in $\mathscr{B}$ such that $A \sim A_{n}$ for each $n \geq 1$. Define a relation $R$ on $\mathscr{B}$ by allowing $(A, B) \in R$ just in case there is some $C \in \mathscr{B}$ with $C \subseteq B$ and $A \sim C$.

THEOREM (CHUAQUI). Let $(X, \mathscr{B})$ be a measurable space and let $\sim$ be a countably additive and refining equivalence relation on $\mathscr{B}$ such that

(1) $X$ is not negligible for ;

(2) the relation $R$ linearly orders the $n$ equivalence classes.

Then there is a unique probability measure $\mu$ on $(X, \mathscr{B})$ such that

(a) $\mu(A)=0$ if and only if $A \in \mathscr{B}$ is negligible for $\sim$;

(b) if $\mu(A) \neq 0$, then $\mu(A)=\mu(B)$ if and only if $A \sim B$.

InDICATION. This is Theorem 2.11 in [3]. Compare also [4].

Call a metric space $X$ universally measurable (u.m.) if for each Borel probability measure $\mu$ on $X$, there is a $\sigma$-compact set $S \subseteq X$ with $\mu(S)=1$. Note that each such $S$ is a Borel set in its completion. Thus, if $S$ is infinite, and $t=t(S)$, then $t=\omega t$.

Each analytic space is u.m. [5, 8.4.1]. The u.m. subsets of an uncountable Polish space $P$ form a $\sigma$-field containing the $\sigma$-field generated by the analytic subsets of $P$.

Our main result applies not only to analytic spaces, but to u.m. spaces in general.

Proposition. Let $X$ be an infinite u.m. space with $t=t(X)$. If $\sec (t)$ is linearly ordered, then $t=\omega t$.

Demonstration. Assume rather that $t<\omega t$. Consider the $\sigma$-field $\mathscr{B}(X)$ under the equivalence relation $\sim$ defined by

$$
A \sim B \Leftrightarrow A, B \text { Borel isomorphic. }
$$

The relation $\sim$ is countably additive and refining. Because $t<\omega t$, we have that $X$ is not negligible. This and the fact that $\sec (t)$ is linearly ordered allows the application of Chuaqui's theorem.

Thus there is a Borel probability measure $\mu$ on $X$ such that $\mu(A)=0$ if and only if $A$ is negligible. Because $X$ is u.m., there exists an infinite $\sigma$-compact $S \subseteq X$ with $\mu(S)=1$. However, $t(S)=\omega t(S) \leq t$, so that each such $S$ is negligible, a contradiction. Q.E.D.

COROLLARY. Under $V=L$, the poset $(A N, \leq)$ is not linearly ordered.

It should be noted that the corollary can be obtained directly from the methods of [1]. In fact, the sets $C d[n]$ defined on p. 16 of [1] are pairwise incomparable.

\section{REFERENCES}

1. D. Cenzer and R. D. Mauldin, Borel equivalence and isomorphism of coanalytic sets, Dissertationes Math. 228 (1984), 1-32.

2. J. P. R. Christensen, Topology and Borel structure, North-Holland and Elsevier, Amsterdam and New York, 1974. 
3. R. Chuaqui, Cardinal algebras and measures invariant under equivalence relations, Trans. Amer. Math. Soc. 142 (1969), 61-79.

4. R. Chuaqui and J. Malitz, Preorderings compatible with probability measures, Trans. Amer. Math. Soc. 279 (1983), 811-824.

5. D. L. Cohn, Measure theory, Birkhäuser, Boston, Mass., 1980.

6. L. Harrington, Analytic determinacy and $0^{\#}$, J. Symbolic Logic 43 (1978), 685-693.

7. N. N. Lusin, Les ensembles analytiques, 2nd ed., Chelsea, New York, 1972.

8. J. Steel, Analytic sets and Borel isomorphism, Fund. Math. 108 (1980), 82-88.

DEPARTMENT OF MATHEMATICS, WESLEyAN UNIVERSity, MidDLETOWN, CONNECTICUT 06457 